\title{
Diabetes and Bone
}

\section{Peter Vestergaard}

Department of Endocrinology and Internal Medicine, Aarhus University Hospital THG, Aarhus, Denmark

\begin{abstract}
Diabetes - both type 1 (T1D) and type 2 (T2D) has profound effects on the skeleton. Bone turnover is reduced, i.e. bone biopsies show a reduced bone formation with reduced mineralisation, and a reduced number of bone cells. Biochemical markers of bone turnover show a reduction in both formation and resoprtion. Bone mineral density (BMD) is reduced in T1D, whereas an increased BMD is seen in T2D. Despite this, an increased risk of hip fractures is seen in both T1D and T2D, the increase in risk of fractures being more pronounced in T1D than in T2D. This discrepancy between BMD and fracture risk along with evidence from animal studies of a reduced bone biomechanical competence in diabetes suggest that the bone tissue is weakened in patients with diabetes. This weakening may be related to glycation of collagen and formation of advanced glycation end-products (AGE), which together with their receptor (RAGE) lead to a decreased activity of the bone cells and thus a reduced turnover and a reduced de-novo formation of bone. The reduced competence despite normal BMD makes the diagnosis of osteoporosis difficult as standard bone scans (DXA) may not truly reflect bone strength. Regarding anti-diabetic treatment, most such drugs improve glucose control and thus reduce the detrimental effects of diabetes on the skeleton. However, the thiazolidinediones (rosiglitazone and pioglitazone) are associated with a decreased BMD and an increased fracture risk through an effect on the stem cells in the bone marrow leading to formation of adipocytes rather than osteoblasts. Anti-resoprtive treatment for osteoporosis seem equally effective in diabetes patients as in non-diabetics despite the reduced bone turnover in diabetes.
\end{abstract}

In conclusion diabetes has many effects on bone and bone-turnover, and more research is needed

Keywords: Diabetes; Osteoporosis; Fracture; Type 1 diabetes; Type 2 diabetes

\section{Introduction}

Diabetes and osteoporosis are both frequent endocrine disorders However, they seem to be interconnected in several ways as demonstrated by recent studies, who have shown an increased risk of fractures [1], a decreased bone mineral density (BMD) in patients with type 1 diabetes (T1D) [2], an increased BMD in patients with type 2 diabetes (T2D) [2], evidence of an impaired bone biomechanical competence [3], and evidence of disrupted bone turnover with a low bone turnover $[4,5]$.

\section{Material and Methods}

The following presents a narrative review of the effects of diabetes on the skeleton.

\section{Review}

Bone turnover: Bone is constantly being formed through the action of osteoblasts and degraded through the action of osteoclasts [6]. Usually formation and resorption (degradation) are tightly coupled. Bone turnover may be assessed through biopsies of bone [7], but may also be assessed more practically through sampling of blood and urine [8]. Patients with diabetes in general have a reduced bone turnover expressed by decreased levels of biochemical markers of bone turnover $[4,5]$.

Formative biochemical markers of bone turnover: Formative markers of bone turnover include osteocalcin, which is a peptide embedded in bone matrix during formation, and alkaline phosphatase, which is an enzyme secreted by the osteoblasts as part of the formation of mineral matrix. PINP is the N-terminal peptide of procollagen type 1 , which is embedded in bone matrix and thus is a formative marker.

Many studies have shown decreases in osteocalcin level of $7 \%$ to $22 \%[4,9-12]$. However, osteocalcin may also be a marker of beta-cell function and not just a marker of bone turnover [13]. For alkaline phosphatase, no study has reported a significant decrease in diabetics compared to controls. One study reported a $7 \%$ nonsignificant decrease in both total alkaline phosphatase and bone-specific alkaline phosphatise [9], while two other studies [11,12] reported small nonsignificant decreases in total alkaline phosphatase of $2 \%$ and $4 \%$, respectively. One study actually reported increased levels of alkaline phosphatase in young adult T1D patients indicative of impaired osteoblast differentiation and maturation, which down-regulated later stages of matrix mineralization [14].

It is remarkable that total alkaline phosphatase was not different since 25-hydroxyvitamin $\mathrm{D}$ tended to be significantly lower in patients with diabetes than in controls [12]. In general low vitamin D may lead to osteomalacia with an increased level of alkaline phosphatase reflecting increased deposition of unmineralised bone. Vitamin D may thus counter high alkaline phosphatase levels [15]. However, it may be that the diabetes countered the effect of the lower vitamin $\mathrm{D}$ to increase alkaline phosphatase by lowering bone turnover and thus alkaline phosphatase per se.

Biochemical markers of bone resorption: Bone resorption may among others be assessed through measurements of N-terminal

Corresponding author: Dr. Peter Vestergaard MD PhD MedSc, The Osteoporosis Clinic, Department of Endocrinology and Internal Medicine (MEA), Aarhus University Hospital THG, Tage Hansens Gade 2, DK-8000 Aarhus C, Denmark Tel: + 45894976 52; Fax: + 45894976 84; E-mail: p-vest@post4.tele.dk

Received July 27, 2011; Accepted August 29, 2011; Published September 16 2011

Citation: Vestergaard P (2011) Diabetes and Bone. J Diabetes Metab S:1 doi:10.4172/2155-6156.S1-001

Copyright: (C) 2011 Vestergaard P. This is an open-access article distributed unde the terms of the Creative Commons Attribution License, which permits unrestricted use, distribution, and reproduction in any medium, provided the original author and source are credited. 
cross links of collagen (NTx) and C-terminal markers of cross-links of collagen (CTX). These cross-links along with urine pyridinoline represents degraded collagen.

Urine NTx has been reported to be markedly and statistically significantly decreased by $66 \%$ in diabetes [11]. A smaller, but still significant, decrease of $14 \%$ has been observed for urine deoxypyridinoline [12]. Regarding CTX, a nonsignificant decrease of around $26 \%$ has been reported in one study [4], while another study reported a significant $31 \%$ decrease in CTX in patients with diabetes [12].

Effects of the changes in bone turnover: It thus seems that bone formation may be decreased ranging from small decreases of $2-7 \%$ for alkaline phosphatase to $7-22 \%$ for osteocalcin. The resorptive markers show an even larger reduction of $14-66 \%$. The larger reduction for resorption as expressed by biochemical markers of bone turnover is in line with the results from a study in healthy college students where an oral glucose load of $75 \mathrm{~g}$ was associated with a $5-10 \%$ reduction in the formative marker P1NP, whereas the resorptive marker CTX decreased by $40-50 \%$ [16]. In theory this imbalance should lead to accumulation of bone and thus an increase in bone mineral density. This accumulation of BMD is actually seen in patients with type 2 diabetes (T2D) [2], whereas patients with type 1 diabetes (T1D) have a decreased BMD [2]. However, in contrast to non-diabetics, where an increase in BMD is associated with a decrease in the risk of fractures [17], a reduction is not seen in patients with diabetes [1,2].

Causes of the decrease in biochemical markers of bone turnover: The decrease in bone turnover may be related to the hyperglycemia since acute ingestion of glucose in healthy non-diabetic subjects leads to a decrease in CTX of around $40-50 \%$ and in P1NP of 5\% after two hours [16]. However, it may be questioned if such a rapid decrease really reflects changes in bone turnover, but rather may reflect extraskeletal alterations in e.g. degradation and excretion of the markers in question. Food intake including ingestion of glucose affects a number of gastrointestinal hormones among other the incretins such as glucagon-like-peptide 1 and 2 (GLP 1 and 2) and glucose-dependent insulinotropic peptide (GIP - also called gastric inhibitory peptide), which reduced bone resorption [18]. GLP 2 seems to dissociate bone resorption and formation [19], which is usually tightly coupled. Nocturnal bone resorption is e.g. suppressed by GLP 2 [18]. Treatment with GLP 2 has actually been shown to increase BMD [20].

Effects on bone turnover assessed by bone biopsies and animal experimental data: Few data are available on histomorphometry in humans with diabetes [5,21]. In general the number of osteoblasts and osteoclasts are reduced in both human $[5,21]$ and animal studies [22-25]. The animal models have included spontaneously diabetic rats [22,23], and streptozotocin-induced diabetic rats [24,25].

In streptozotocin-induced diabetes in rats, a study of mandibular bone, showed that the number of osteoclasts were reduced [26] in accordance with the observations of reduced bone resorption from studies of biochemical markers [11]. In Spontaneously diabetic BB/ OK (F60/61) rats, mineralization lag time may be prolonged from around 1 day to around 15 days and mineralizing surfaces may decrease from $32 \%$ to $9 \%$ in poorly controlled diabetic rats [22]. Upon administration of insulin, these changes are reversed [22]. This decrease in mineralization and thus bone formation is far greater than what should be expected from the bone formative biochemical markers [4,9-12]. The quality of the newly formed bone may also be questionable [27]. In addition to the changes described above, a recent study on alveolar bone in streptozotocin-induced diabetes in Wistar rats showed that bone quality was significantly deteriorated as evaluated by micro CT [26]. A study on osteoclast function using a mice model of streptozotocin-induced diabetes showed impaired osteoclast function [28]. This study concluded that the delayed bone formation in the diabetic mice may have resulted from an impairment of cartilage resorption. The study reported that $80 \%$ of the osteoclasts in the callus of the experimental fractures were derived from bone marrow and that the sizes of the osteoclasts as well as the resorption pits formed were significantly smaller in the diabetic mice than in nondiabetic mice [28]. Transcript analyses using RNA isolated by laser capture microdissection showed that the expression of DC-STAMP, a putative pivotal gene for osteoclast fusion, was decreased in osteoclasts from diabetic mice [28]. Since the sustainability of osteoclast function depends on the controlled renewal of multinuclear osteoclasts, impaired osteoclast function in diabetes may contribute to decreased cartilage resorption and delayed endochondral ossification [28].

\section{Osteocalcin, diabetes, and bone}

Animal experimental studies have revealed a link between the bone turnover marker osteocalcin and type 2 diabetes [13]. Mice lacking osteocalcin have decreased insulin levels and increased blood glucose levels [13]. This may however not directly be translated into clinical practice, as patients with diabetes have a number of other metabolic disturbances as previously mentioned, and these bone metabolic distrubances may per se affect osteocalcin levels. The lower insulin levels in the knock-out mice mimic a state which is intermediate to T1D (absolute insulinopenia) and T2D (insuficient insulin secretion for the requirements) [13]. It may thus both mimic early stages of T1D where insulin secretion is declining and late stages of T2D where the initial increase in insulin secretion is followed by a decrease resulting from declining beta-cell function. Although osteocalcin may affect insulin secretion [29] it is not entirely clear if the opposite is the case. In streptozotocin induced diabetes in rats, serum osteocalcin decreases, and histomorphometry shows decreased bone remodeling [24].

In patients with diabetes a cross sectional study has shown decreased levels of osteocalcin in patients with T1D and increased levels in patients with T2D irrespective of whether these were treated with oral hypoglycemic agents or insulin compared to control subjects [30]. However, this could not be confirmed in another study, who reported normal levels of osteocalcin in patients with T1D and decreased levels in T2D compared with normal controls [31]. A further study in male patients with T2D also showed reduced osteocalcin levels, whereas no differences between patients and controls were evident for sex steroid status and CTX levels [4]. The findings of the latter two studies [4,31] are more in line with what should be expected from histomorphometric findings of a decreased bone formation [21]. Upon correction of poorly regulated blood glucose levels, serum osteocalcin has been shown to increase [32].

In patients with T1D serum osteocalcin levels have been shown to be lower in patients with complications (retinopathy and/or proteurinuria) than in patients with diabetes without complications $[31,33]$. In newborns to mothers with diabetes, umbilical cord osteocalcin levels have been shown to be decreased [34].

Due to the usually close coupling between bone formation and resorption, other markers of bone formation such as P1NP and resoprtion such as CTX are also decreased [16]. 


\section{Advanced glycation end products (AGE) and their receptor (RAGE)}

Besides the direct effects on bone turnover and bone density, bone biomechanical competence may also be altered in diabetes through glycation of other proteins besides haemoglobin (examplified by HbA1C). The glycation may alter the strength of the bone matrix thus leading to an increased brittleness of bone that is not directly reflected in BMD.

The prime glycation end product is pentosidine [35], but a number of other advanced glycation end products (AGE) and their receptor (RAGE) may be involved in the development of arteriosclerosis and diabetic microangiopathy [36]. The AGE-RAGE interaction activates nuclear factor $\mathrm{kB}(\mathrm{NF}-\mathrm{kB})$, which interacts with cytokines, growth factors and adhesion molecules, all of which may contribute to osteoporosis. Glycation may reduce cross-linking in collagen and thus reduce mineralisation and bone biomechanical competence [35]. It also interacts with osteoblast functioning thus reducing bone formation [37]. This may also affect processes vital to bone turnover such as glucosylation of skeletal collagen leading to decreased strength of the collagen [38] and thus of the bone and abnormal resorption and formation of bone [35,37,39-42]. AGE may decrease osteoclast activity [43], and decreased osteoclast activity would lead to less bone resorption. Thus, the net effect is not fully understood. Osteoblastic cells from rats, who were cultured on AGE-modified type 1 collagen, showed a reduced secretion of alkaline phosphatase (ALP) and osteocalcin. Also formation of bone nodules was reduced $[44,45]$.

Furthermore, AGE may affect osteoblastic function by stimulating osteoblast apoptosis via the MAP kinase and cytosolic apoptotic pathways as demonstrated in a study using cell culture and mice [46]. This was further supported and expanded by a study using cell cultures that reported that AGE significantly decreased osteoblast proliferation, alkaline phosphatase activity and type 1 collagen production, while increasing osteoblastic apoptosis and reactive oxygen species production [47]. These effects were completely reverted by low doses of bisphosphonates [47] lending support to a positive effect of these drugs despite a low bone turnover I diabetes. These studies are thus in line with the observations of a decrease in formative bone markers. Bone collagen from rats with streptozotocin-induced diabetes had a higher accumulation of AGE, which was associated with a reduced BMD [44]. This may thus explain the reduced BMD in T1D but not the increased $\mathrm{BMD}$ in $\mathrm{T} 2 \mathrm{D}$.

Patients with T1D are insulinopenic and patients with T2D may have increased levels of insulin. In general serum insulin levels are positively correlated with BMD [48], and this may explain why BMD in general is higher in T1D than in T2D [2]. The increased insulin levels in T2D may also be associated with increased levels of insuline-likegrowth factor (IGF), which may also be bone anabolic [5].

\section{Negative calcium balance}

Early studies pointed at an increased calcium excretion in the urine from calciuric effects of the high blood sugar [49]. This leads to a loss of calcium from the skeleton with a decrease in the bone mineral density (BMD) [49] and an increased risk of fractures [2]. A direct correlation has been shown between the blood sugar levels and the excretion of calcium in the urine $[49,50]$, and the loss of calcium from the forearm may amount to as much as $10 \%$ within the first 5 years after the diagnosis of diabetes [49].

Besides the effects of high blood glucose on calcium excretion in the urine, the blood pressure also has effects on urine calcium excretion. The higher the blood pressure, the greater the loss of calcium is in the urine [51]. This may represent a hitherto somewhat overlooked risk factor for osteoporosis as hypertension is also associated with a decreased BMD, and thus an increased risk of fractures [51]. In diabetes, hypertension may thus contribute further to the negative calcium balance [52].

\section{Bone mineral density}

$\mathrm{BMD}$ is reduced by approximately $0.2 \mathrm{Z}$-scores in the hip and spine in T1D, while it is increased by approximately $0.3-0.4 \mathrm{Z}$-scores in the hip and spine in T2D [2]. In theory this should translate into an increase in hip fractures of $\mathrm{RR}=1.4$ in T1D and a decrease $\mathrm{RR}=0.8$ of in $\mathrm{T} 2 \mathrm{D}$ [2]. This is in stark contrast to the observed RR for hip fractures of 6.2 in T1D and 1.7 T2D [2] pointing at a weakening of bone biomechanical competence in diabetes [3]. In diabetes [2] as well as in non-diabetics [53] a positive relationship exists between body mass index (BMI) and $\mathrm{BMD}$, to some extent explaining the differences between the often normal to underweight T1D patients and the obese T2D patients [2] No differences in BMD seemed present between T1D and T2D after adjustment for body weight [2]. It thus seems that the effect of the underlying diabetic condition may be the same and probably linked to glucose levels.

\section{Fracture risk}

In T1D an increased risk of hip fractures was seen [2]. However, for other fracture types the number of studies was too scarce for a metaanalysis [2] although most studies revealed a trend towards an increase [2]. In T2D an increased risk of hip, foot, and perhaps overall risk of fractures was seen $[1,2]$, while no increase in spine, forearm, or ankle fractures was seen $[1,2]$. It thus seems that the hip is the fracture site with the most consistent increase in fracture risk in both T1D and T2D. However, little is known for other skeletal sites.

From one study it seems that the increase in risk of fractures in T2D is seen early after diagnosis followed by a decline to the same levels as in the background population [54]. The reasons for this are not known but may signal an effect of the increased calcium loss in the urine with high blood glucose levels as it is in line with early studies suggesting a marked negative calcium balance that is corrected once better metabolic control is obtained, which also reflected in an increase in forearm bone mineral content $[49,50]$. However, an effect on bone biomechanical competence cannot be excluded with better control of blood glucose levels.

The increase in risk of fractures did not to any mjor degree seem linked to complications to the diabetes, i.e. was not the result of falls from impaired eye-sight [54], decreased propioception from neuropathy [54], or falls related to accidents stemming from hypoglycemia [55]. It may thus be the effects of the underlying alterations in bone competence that were responsible for the increase in risk of fractures.

The overweight and obesity seen in patients with T2D may thus merely protect against the loss of bone [2] and to some degree ameliorate the increase in risk of fractures as also seen in non-diabetics [53].

\section{Effects of treatment}

Effects of drugs against diabetes: In animal models, improvement of diabetes control has resulted in reversal of the histomorphometric changes induced by diabetes [22]. Observational studies have pointed at a reversal of e.g. the loss of calcium in the urine and an improvement of BMD when blood glucose levels are better controlled 
[49]. Observational studies have also suggested that the excess risk of fractures may decline with time since diagnosis of diabetes, and thus improved metabolic control [54]. However, one observational study failed to show an association between $\mathrm{HbA1C}$ and BMD [2]. This should be interpreted with caution: $\mathrm{HbA1C}$ reflects blood glucose levels within the last 6-8 weeks, and bone turnover is a process that takes much longer than 6-8 weeks. Also BMD may not adequately reflect bone biomechanical competence in patients with diabetes.

In general drugs against diabetes improve metabolic control, and should thus be expected to be able to prevent osteoporosis. Insulin, the sulphonylureas, and metformin seem associated with a decrease in the risk of fractures or a trend towards a decrease [55] probably related to better metabolic control of diabetes. Metformin has also been shown to have positive effects on bone turnover by improving metabolic control [56]. Recently published results from rodent models suggest a positive effect of exenatide on bone, but there are no clinical studies [57].

Thiazolidinediones (TZD): TZDs act by improving insulin sensitivity through activation of the nuclear receptor, peroxisome proliferator-activated receptor $\gamma$ (PPAR- $\gamma$ ) [58]. The TZDs affect the differentiation of mesenchymal stem cells [59]. Normally the common mesenchymal progenitor stem cell can differentiate into among others osteoblasts and bone marrow adipocytes [60]. TZDs increase adipogenesis at the expense of osteoblasts, leading to bone loss [61,62]. Other effects by PPAR $\gamma$ agonists include decreased circulating IGF-I concentrations [63], and a decrease in oestrogens levels, as treatment has been shown to affect the synthesis of sex steroids by inhibiting the aromatase pathway which is the main source for oestrogen in postmenopausal women [64].

Mice treated with troglitazone show an increased marrow concentration of adipocytes [59], whereas the ratio of osteoblasts to osteoclasts decreases causing bone loss in mice treated with rosiglitazone [62]. Similarly, treatment with rosiglitazone has been shown to decrease BMD in mice [65-67]. Concomitantly with decreased BMD, treatment may change bone morphology, as a decreased trabecular number and an increase in trabecular spacing has been reported [67]. In human cell models [68] the effect of rosiglitazone on adipogeneis has also been confirmed. Besides the effects on osteoblasts, TZDs may also have an effect on the osteoclasts [69] with an increased osteoclast differentiation due to the PPAR agonistic effect of rosiglitazone. The clinical effects of this are not completely understood.

Two randomised controlled trial (RCT) have shown decreased levels of biochemical markers of bone formation in humans treated with rosiglitazone, indicating a decreased activity of osteoblastic cells [9]. In a group of 82 postmenopausal women, 12 weeks of treatment with rosiglitazone $4 \mathrm{mg} / \mathrm{d}$ caused a $21 \%$ decrease in plasma levels of bone-specific alkaline phosphatase, compared with placebo $(\mathrm{p}<0.05)$ [9]. Similarly, in a RCT including 50 postmenopausal women without diabetes or osteoporosis, 14 weeks of treatment with rosiglitazone 8 $\mathrm{mg} / \mathrm{d}$ caused an approximately $10 \%(\mathrm{p}<0.05)$ decrease in biochemical markers of bone formation (osteocalcin and procollagen type I $\mathrm{N}$-terminal propeptide), while markers of bone resorption did not change in response to treatment [70]. Moreover, compared with placebo, rosiglitazone caused a $1.7 \%$ (95\% CI 0.6 to $2.7, \mathrm{p}<0.01$ ) decrease in BMD at the total hip. BMD at the lumbar spine decreased as well but did not differ significantly between groups $(1.0 \%, 95 \% \mathrm{CI}$ -0.2 to $2.3, p=0.13$ ) at the end of treatment [70]. The findings from the RCTs are in line with the results from a previous cohort study showing that treatment with glitazones is associated with approximately $50 \%$ increased annualized rate of bone loss in elderly diabetic women, whereas no effects were found in men [71]. However, in elderly men with T2DM significantly higher annualized bone loss rates have been found in those treated with glitazones $(-1.22 \pm 1.3 \%, \mathrm{n}=32)$ compared with those who were not $(-0.20 \pm 1.25 \%, \mathrm{n}=128)$ [72]. The fact that bone turnover is often increased in elderly women compared with elderly men may augment the consequences of reduced osteoblastic activity in response to treatment with TZDs, which may explain that the effects of TZDs on BMD is more consistently present in women than in men.

Most important, treatment with TZDs seems to increase fracture risk. According to the results from the ADOPT study, risk of fracture is approximately doubled in users of rosiglitazone compared with risk of fracture in users of either metformin or glyburide [73]. In the ADOPT trial, 4,360 (42\% women) participants with a mean age of 57 years were followed for a median of 4 years. The study was a RCT designed to evaluate rosiglitazone, metformin, and glyburide as initial treatment for recently diagnosed T2DM. The primary outcome was time to monotherapy failure as determined by fasting glucose. Review of adverse event reports revealed that the proportion of women reporting a fracture was $9.3 \%$ for rosiglitazone, 5.1\% for metformin, and 3.5\% for glyburide, corresponding to an approximate relative risk (RR) of 2.3 (95\% CI 1.6-3.4) for rosiglitazone versus the other treatments combined. However, although fractures were similarly distributed across treatment groups in male participants, the increase in risk of fracture did not reach statistical significance (RR 1.2; 95\% CI, 0.8-1.8 for rosiglitazone versus the other treatments combined). Similar to treatment with rosiglitazone, treatment with pioglitazone has been reported to increase risk of fracture [74], and the increase in risk of fractures was similar for rosiglitazone and pioglitazone [74].

In terms of fracture risk it is an important finding that TZDs as the only class of drugs against diabetes seems associated with an increased risk of fractures, whereas all other classes of drugs seeming either are not associated with risk of fractures or are associated with a small decrease in risk of fractures probably linked to the glucose-lowering effect and thus improvement of the otherwise negative effects on bone of hyperglycaemia [55].

Regarding bone density measurements in patients being started or currently managed with TZD, no consensus exists. Also no consensus exists as to the best way to manage patients with decreased BMD receiving TZDs. At present care should be taken not to prescribe TZDs to patients at high risk of fractures, i.e. patients with osteoporosis (T-score <-2.5 at any site) and significant risk factors for fractures. The potential benefit of TZDs in terms of improved diabetes control (and thus also indirectly potentially better bone turnover) should be carefully weighed against the risk of fractures, as the risk of fractures seems increased even in the presence of improved diabetes control [73].

In patients which have a low a priori risk of fractures (men and women below the age of approximately 50 years), BMD measurements by DXA may not be particularly indicated if no other risk factors are present, whereas in postmenopausal women and men above the age of 65 years, the prevalence of osteoporosis is so high that DXA scannings may be indicated [75]. Depending on the level of bone density, DXA scans may be repeated after 2-3 years [75]. However, this may represent a rather large cost in comparison with the gains obtained. TZD therapy thus needs to be carefully considered in patients at high risk of fractures and osteoporosis.

Serum and urine markers of bone turnover are very variable and do thus not have a place in the considerations regarding risk of osteoporosis [76]. 
Weight loss: A special feature is that weight loss is encouraged in obese patients with T2D. A randomised controlled trial in overweight patients with T2D showed that weight loss was associated with a decrease in BMD $(0.9 \%$ decrease in total body BMD over 12 month with non-significant decreases in spine and femur BMD) [77], and that exercise training seemed to prevent the loss of BMD [77]. In patients treated by gastric by-pass, which is used for weight reduction in morbidly obese patients with T2D, a decrease is seen in BMD [78].

\section{Conventional treatment of osteoporosis with anti-resoprtive drugs}

Antiresorptive drugs: These include the bisphosphonates and the selective estrogen receptor modulators (SERMs) [79]. In theory these may present a special problem as bone turnover is low in diabetes. Two studies have shown improvements in BMD with the bisphosphonate alendronate over placebo [80,81]. The paper by Keegan et al. was a post hoc analysis of a randomized trial [81]. The paper by Dagdelen et al. was an observational study without a placebo group [80]. In this study, the authors found that diabetic patients on alendronate lost bone compared to non-diabetic patients on alendronate. The study is difficult to interpret since we do not know how much bone the diabetic patients would have lost on placebo [80]. However, no fracture data are available. It may thus be concluded that the decrease in bone turnover does not lead to decreases in BMD, however, it is not possible to deduct if the quality of the newly formed bone is adequate. An observational study suggested that anti-resoprtive drugs including the bisphosphonates were equally effective in patients with diabetes as in patients without [82]. However, it may be that the diabetes in these subjects was well-controlled. What happens in patients with poorly controlled diabetes is not known. Further studies are thus needed.

A post hoc analysis of a raloxifene trial identified reduced vertebral fracture risk compared to placebo in participants with diabetes [83]. However, this study only included patients with T2D and the number of patients was very limited [83]. Strontium ranelate is also effective against osteoporosis [84], however for this compound no specific studies on the effects in patients with diabetes are available.

Anabolic drugs: This class of drugs include parathyroid hormone and analogues $[85,86]$. In theory these drugs may pose an advantage due to the decreased bone turnover in patients with diabetes, but no clinical studies are available. However, one study have indicated that teriparatide may perhaps have a limited actue adverse effect on insulin resistance [87]. Further studies are thus needed.

\section{Conclusion}

In conclusion bone mineral density is decreased and hip fracture risk increased in patients with T1D, while in patients with T2D hip fracture risk is increased although to a lesser degree than in T1D. However, in T2D patients bone mineral density is increased. This points at a decreased bone quality in patients with diabetes. Patients with diabetes should be treated against osteoporosis when indicated. It is important to be aware that patients with diabetes do seem to tolerate anti-resoprtive drugs including the bisphosphonates despite having biochemical signs of reduced bone turnover.

\section{References}

1. Janghorbani M, van Dam R, Willett W, Hu F (2007) Systematic review of type 1 and type 2 diabetes mellitus and risk of fracture. Am J Epidemiol 166: 495-505.

2. Vestergaard $P(2007)$ Discrepancies in bone mineral density and fracture risk in patients with type 1 and type 2 diabetes-a meta-analysis. Osteoporos Int 18: $427-444$
3. Saito M, Marumo K (2010) Collagen cross-links as a determinant of bone quality: a possible explanation for bone fragility in aging, osteoporosis, and diabetes mellitus. Osteoporos Int 21: 195-214.

4. Achemlal L, Tellal S, Rkiouak F, Nouijai A, Bezza A, et al. (2005) Bone metabolism in male patients with type 2 diabetes. Clin Rheumatol. 24: 493-496.

5. Bouillon R (1992) Diabetic bone disease. Low turnover osteoporosis related to decreased IGF-I production. Verh K Acad Geneeskd Belg 54: 365-91 discussion 391-392.

6. Delmas P (1995) Biochemical markers for the assessment of bone turnover In Osteoporosis: etiology, diagnosis and management. Riggs B, Melton III L (Eds.) pp. 319-333.

7. Eriksen E, Steiniche T, Mosekilde L, Melsen F (1989) Histomorphometric analysis of bone in metabolic bone disease. Endocrinol Metab Clin North Am 18: $919-954$

8. Camacho PM, Lopez NA (2008) Use of biochemical markers of bone turnove in the management of postmenopausal osteoporosis. Clin Chem Lab Med 46 1345-1357.

9. Berberoglu Z, Gursoy A, bayraktar N, Yazici A, Tutuncu N, et al. (2007) Rosiglitazone decreases serum bone specific alkaline phosphatase activity in postmenopausal diabetic women. J Clin Endocrinol Metab 92: 3523-3530.

10. Kindblom JM, Ohlsson C, Ljunggren O, Karlsson MK, Tivesten A, et al. (2009) Plasma Osteocalcin is Inversely Related to Fat Mass and Plasma Glucose in Elderly Swedish Men. J Bone Miner Res 24: 867-874.

11. Akin O, Gol K, Akturk M, Erkaya S (2003) Evaluation of bone turnover in postmenopausal patients with type 2 diabetes mellitus using biochemical markers and bone mineral density measurements. Gynecol Endocrinol 17: 1929.

12. Gerdhem P, Isaksson A, Akesson K, Obrant K (2005) Increased bone density and decreased bone turnover, but no evident alteration of fracture susceptibility in elderly women with diabetes mellitus. Osteoporos Int 16: 1506-1512.

13. Lee N, Sowa H, Hinoi E, Ferron M, Ahn J, et al. (2007) Endocrine regulation of energy metabolism by the skeleton. Cell 130: 456-469.

14. Massé PG, Pacifique MB, Tranchant CC, Arjmandi BH, Ericson KL, et al. (2010) Bone metabolic abnormalities associated with well-controlled type diabetes (IDDM) in young adult women: a disease complication often ignored or neglected. J Am Coll Nutr 29: 419-429.

15. Hahn T, Halstead L (1979) Anticonvulsant drug-induced osteomalacia: alterations in mineral metabolism and response to vitamin D3 administration. Calcif Tissue Int 27: 13-18.

16. Clowes J, Allen H, Prentis D, Eastell R, Blumsohn A (2003) Octreotide Abolishes the Acute Decrease in Bone Turnover in Response to Oral Glucose. J Clin Endocrinol Metab 88: 4867-4873.

17. Marshall D, Johnell O, Wedel H (1996) Meta-analysis of how well measures of bone mineral density predict occurrence of osteoporotic fractures. BMJ 312 : 1254-1259.

18. Henriksen DB, Alexandersen P, Byrjalsen I, Hartmann B, Bone HG, et al (2004) Reduction of nocturnal rise in bone resorption by subcutaneous GLP-2. Bone 34: 140-147.

19. Henriksen DB, Alexandersen P, Hartmann B, Adrian CL, Byrjalsen I, et al (2007) Disassociation of bone resorption and formation by GLP-2: a 14-day study in healthy postmenopausal women. Bone 40: 723-729.

20. Henriksen DB, Alexandersen P, Hartmann B, Adrian $\mathrm{CL}$, Byrjalsen I, et al (2009) Four-month treatment with GLP-2 significantly increases hip BMD a randomized, placebo-controlled, dose-ranging study in postmenopausal women with low BMD. Bone 45: 833-842.

21. Leite Duarte M, da Silva R (1996) [Histomorphometric analysis of the bone tissue in patients with non-insulin-dependent diabetes (DMNID)]. Rev Hosp Clin Fac Med Sao Paulo 51: 7-11.

22. Follak N, Kl”ting I, Wolf E, Merk H (2004) Improving metabolic control reverses the histomorphometric and biomechanical abnormalities of an experimentally induced bone deficit in spontaneously diabetic rats. Calcif Tissue Int 74: 551 560

23. Fujii H, Hamada Y, Fukagawa M (2008) Bone formation in spontaneously diabetic Torii-newly established model of non-obese type 2 diabetes rats. Bone 42: $372-379$. 
24. Glajchen N, Epstein S, Ismail F, Thomas S, Fallon M, et al. (1988) Bone mineral metabolism in experimental diabetes mellitus: osteocalcin as a measure of bone remodeling. Endocrinology 123: 290-295.

25. Hamada Y, Kitazawa S, Kitazawa R, Fujii H, Kasuga M, et al. (2007) Histomorphometric analysis of diabetic osteopenia in streptozotocin-induced diabetic mice: a possible role of oxidative stress. Bone 40: 1408-1414

26. Abbassy MA, Watari I, Soma K (2010) The effect of diabetes mellitus on rat mandibular bone formation and microarchitecture. Eur J Oral Sci 118: 364-369.

27. Verhaeghe J, Suiker AM, Einhorn TA, Geusens P, Visser WJ, et al. (1994) Brittle bones in spontaneously diabetic female rats cannot be predicted by bone mineral measurements: studies in diabetic and ovariectomized rats. $\mathrm{J}$ Bone Miner Res 9: 1657-1667.

28. Kasahara T, Imai S, Kojima H, Katagi M, Kimura H, et al. (2010) Malfunction of bone marrow-derived osteoclasts and the delay of bone fracture healing in diabetic mice. Bone 47: 617-625.

29. Ferron M, Hinoi E, Karsenty G, Ducy P (2008) Osteocalcin differentially regulates beta cell and adipocyte gene expression and affects the development of metabolic diseases in wild-type mice. PNAS 105: 5266-5270.

30. Cantini F, Arcangeli A, Bellandi F, Pedone T, Villani G, et al. (1992) [Serum osteocalcin and diabetes mellitus. A study of 98 patients]. Minerva Med 83: 129-133.

31. Pietschmann P, Schernthaner G, Woloszczuk W (1988) Serum osteocalcin levels in diabetes mellitus: analysis of the type of diabetes and microvascular complications. Diabetologia 31: 892-895.

32. Sayinalp S, Gedik O, Koray Z (1995) Increasing serum osteocalcin after glycemic control in diabetic men. Calcif Tissue Int 57: 422-425.

33. Pasaoglu H, Kumandas S, Kelestimur F (1995) Serum osteocalcin levels in type I diabetes mellitus. Turk J Pediatr 37: 323-329.

34. Verhaeghe J, van Herck E, Bouillon R (1995) Umbilical cord osteocalcin in normal pregnancies and pregnancies complicated by fetal growth retardation or diabetes mellitus. Biol Neonate 68: 377-383.

35. Saito M, Fujii K, Soshi S, Tanaka T (2006) Reductions in degree of mineralization and enzymatic collagen cross-links and increases in glycationinduced pentosidine in the femoral neck cortex in cases of femoral neck fracture. Osteoporos Int 17: 986-995.

36. Yamagishi S, Nakamura K, Inoue H (2005) Possible participation of advanced glycation end products in the pathogenesis of osteoporosis in diabetic patients. Medical Hypotheses 65: 1013-1015.

37. Hein G, Weiss C, Lehmann G, Niwa T, Stein G, et al. (2006) Advanced glycation end product modification of bone proteins and bone remodelling: hypothesis and preliminary immunohistochemical findings. Ann Rheum Dis 65: 101-104.

38. Viguet-Carrin S, Garnero P, Delmas P (2006) The role of collagen in bone strength. Osteoporos Int 17: 319-336.

39. Hein G, Wiegand R, Lehmann G, Stein G, Franke S (2003) Advanced glycation end-products pentosidine and $\mathrm{N}$ epsilon-carboxymethyllysine are elevated in serum of patients with osteoporosis. Rheumatology (Oxford) 42: 1242-1246.

40. Odetti P, Rossi S, Monacelli F, Poggi A, Cirnigliaro M, et al. (2005) Advanced glycation end products and bone loss during aging. Ann N Y Acad Sci 1043: 710-717.

41. Miyata T, Notoya K, Yoshida K, Horie K, Maeda K, et al. (1997) Advanced glycation end products enhance osteoclast-induced bone resorption in cultured mouse unfractionated bone cells and in rats implanted subcutaneously with devitalized bone particles. J Am Soc Nephrol 8: 260-270.

42. Miyata T, Kawai R, Taketomi S, Sprague S (1996) Possible involvement of advanced glycation end-products in bone resorption. Nephrol Dial Transplant 11 Suppl 5: 54-57.

43. Valcourt U, Merle B, Gineyts E, Viguet-Carrin S, Delmas PD, et al. (2007) Non-enzymatic glycation of bone collagen modifies osteoclastic activity and differentiation. J Biol Chem 282: 5691-5703.

44. Katayama Y, Akatsu T, Yamamoto M, Kugai N, Nagata N (1996) Role of nonenzymatic glycosylation of type I collagen in diabetic osteopenia. J Bone Miner Res 11: 931-937

45. Katayama Y, Celic S, Nagata N, Martin TJ, Findlay DM (1997) Nonenzymatic glycation of type I collagen modifies interaction with UMR 201-10B preosteoblastic cells. Bone 21: 237-242.
46. Alikhani M, Alikhani Z, Boyd C, MacLellan CM, Raptis M, et al. (2007) Advanced glycation end products stimulate osteoblast apoptosis via the MAP kinase and cytosolic apoptotic pathways. Bone 40: 345-353.

47. Gangoiti MV, Cortizo AM, Arnol V, Felice JI, McCarthy AD (2008) Opposing effects of bisphosphonates and advanced glycation end-products on osteoblastic cells. Eur J Pharmacol 600: 140-147.

48. Reid I, Evans M, Cooper G, Ames R, Stapleton J (1993) Circulating insulin levels are related to bone density in normal postmenopausal women. Am J Physiol 265: E655-E659.

49. McNair P, Madsbad S, Christensen M, Christiansen C, Faber O, et al. (1979) Bone mineral loss in insulin-treated diabetes mellitus: studies on pathogenesis. Acta Endocrinol 90: 463-472.

50. McNair P, Madsbad S, Christiansen C, Christensen M, Faber O, et al. (1979) Bone loss in diabetes: effects of metabolic state. Diabetologia 17: 283-286.

51. Cappuccio F, Meilahn E, Zmuda J, Cauley J (1999) High blood pressure and bone-mineral loss in elderly white women: a prospective study. Study of Osteoporotic Fractures Research Group. Lancet 354: 971-975.

52. Strazzullo P, Galletti F, Cirillo M, Siani A, Nunziata V, et al. (1986) Altered extracellular calcium homoeostasis in essential hypertension: a consequence of abnormal cell calcium handling. Clin Sci (Lond) 71: 239-244.

53. De Laet C, Kanis J, Oden A, Johanson H, Johnell O, et al. (2005) Body mass index as a predictor of fracture risk: A meta-analysis. Osteoporosis Int 16 : 1330-1338.

54. Vestergaard P, Rejnmark L, Mosekilde L (2009) Diabetes and its complications and their relationship with risk of fractures in type 1 and 2 diabetes. Calcif Tissue Int 84: 45-55.

55. Vestergaard $P$, Rejnmark $L$, Mosekilde $L$ (2005) Relative fracture risk in patients with diabetes mellitus, and the impact of insulin and oral antidiabetic medication on relative fracture risk. Diabetologia 48: 1292-1299.

56. Zhen D, Chen $Y$, Tang $X$ (2009) Metformin reverses the deleterious effects of high glucose on osteoblast function. J Diabetes Complications 24: 334-44.

57. Nuche-Berenguer B, Moreno P, Portal-Nuñez S, Dapía S, Esbrit P, et al. (2010) Exendin-4 exerts osteogenic actions in insulin-resistant and type 2 diabetic states. Regul Pept 159: 61-66.

58. Yki-Jarvinen H (2004) Thiazolidinediones. N Engl J Med 351: 1106-1118.

59. Tornvig L, Mosekilde L, Justesen J, Falk E, Kassem M (2001) Troglitazone treatment increases bone marrow adipose tissue volume but does not affect trabecular bone volume in mice. Calcif Tissue Int 69: 46-50.

60. Nuttall M, Patton A, Olivera D, Nadeau D, Gowen M (1998) Human trabecula bone cells are able to express both osteoblastic and adipocytic phenotype: implications for osteopenic disorders. J Bone Mineral Res 13: 371-382.

61. Lecka-Czernik B, Moerman E, Grant D, Lehmann J, Manolagas S, et al. (2002) Divergent effects of selective peroxisome proliferator-activated receptorgamma ligands on adipocyte versus osteoblast differentiation. Endocrinology 143: $2376-2384$

62. Ali A, Weinstein R, Stewart S, Parfitt A, Manolagas S, et al. (2005) Rosiglitazone causes bone loss in mice by suppressing osteoblast differentiation and bone formation. Endocrinology 146: 1226-1235.

63. Lecka-Czernik B, Ackert-Bicknell C, Adamo M, Marmolejos V, Churchill G, et al (2007) Activation of peroxisome prolioferator-activated receptor gamme (PPAR gamma) by rosiglitazone suppresses components of the insulin-like growth factor regulatory system in vitro and in vivo. Endocrinology 148: 903-911.

64. Rubin G, Zhao Y, Kalus A, Simpson E (2005) Peroxisome proliferator-activated receptor gamma ligands inhibit estrogen biosynthesis in human breast adipose tissue: possible implications for breast cancer therapy. Cancer Res 60: 1604 1608 .

65. Lazarenko O, Rzonca S, Hogue W, Swain F, Suva L, et al. (2007) Rosiglitazone induces decreases in bone mass and strength that are reminiscent of aged bone. Endocrinology 148: 2669-2680.

66. Soroceanu M, Miao D, Bai X, Su H, Goltzman D, et al. (2004) Rosiglitazone impacts negatively on bone by promoting osteoblast/osteocyte apoptosis. J Endocrinol 183: 203-216

67. Rzonca S, Suva L, Gaddy D, Montague D, Lecka-Czernik B (2004) Bone is a target for the antidiabetic compound rosiglitazone. Endocrinology 145: 401-406. 
68. Benvenuti S, Cellai I, Luciani P, Deledda C, Baglioni S, Giuliani et al. (2007) Rosiglitazone stimulates adipogenesis and decreases osteoblastogenesis in human mesenchymal stem cells. J Endocrinol Invest 30: RC26-30.

69. Wan Y, Chong L, Evans RM (2007) PPAR-gamma regulates osteoclastogenesis in mice. Nat Med 13: 1496-1503.

70. Grey A, Bolland M, Gamble G, Wattie D, Horne A, Davidson J, Reid I (2007) The peroxisome-proliferator-activated receptor-gamma agonist rosiglitazone decreases bone formation and bone mineral density in healthy postmenopausa women: a randomised controlled trial. J Clin Endocrinol Metab 92: 1305-1310.

71. Schwartz A, Sellmeyer D, Vittinghoff E, Palermo L, Lecka-Czernik B, et al (2006) Thiazolidinedione use and bone loss in older diabetic adults. J Clin Endocrinol Metab 91: 3349-3354.

72. Yaturu S, Bryant B, Jain S (2007) Thiazolidinedione treatment decreases bone mineral density in type 2 diabetic men. Diabetes Care 30: 1574-1576.

73. Kahn S, Haffner S, Heise M, Herman W, Holman R, et al. (2006) Glycemic durability of rosiglitazone, metformin, or glyburide monotherapy. N Engl J Med 355: $2427-2443$

74. Meier C, Kraenzlin ME, Bodmer M, Jick SS, Jick H, et al. (2008) Use of Thiazolidinediones and Fracture Risk. Archives of Internal Medicine 168: 820825.

75. Leib E, Lewiecki E, Brinkley N, Hamdy R (2004) Official Positions of the International Society for Clinical Densitometry. J Clin Densitom 7: 1-6.

76. Vestergaard P, Hermann A, Gram J, Jensen L, Eiken P, et al. (2001) Evaluation of methods for prediction of bone mineral density by clinical and biochemical variables in perimenopausal women. Maturitas 40: 211-220.

77. Daly RM, Dunstan DW, Owen N, Jolley D, Shaw JE, et al. (2005) Does highintensity resistance training maintain bone mass during moderate weight loss in older overweight adults with type 2 diabetes? Osteoporos Int 16: 1703-1712.

78. Giusti V, Gasteyger C, Suter M, Heraief E, Gaillard RC, Burckhardt P (2005) Gastric banding induces negative bone remodelling in the absence of secondary hyperparathyroidism: potential role of serum $\mathrm{C}$ telopeptides for follow-up. Int $J$ Obes (Lond) 29: 1429-1435.

79. Mosekilde L, Vestergaard P, Langdahl B (2006) Fracture prevention in postmenopausal women. Evidence Based Medicine 1543-1560.

80. Dagdelen S, Sener D, Bayraktar M (2007) Influence of type 2 diabetes mellitus on bone mineral density response to bisphosphonates in late postmenopausal osteoporosis. Adv Ther 24: 1314-1320.

81. Keegan THM, Schwartz AV, Bauer DC, Sellmeyer DE, Kelsey JL (2004) Effect of alendronate on bone mineral density and biochemical markers of bone turnover in type 2 diabetic women: the fracture intervention trial. Diabetes Care 27: $1547-1553$

82. Vestergaard P, Rejnmark L, Mosekilde L (2011) Are antiresorptive drugs effective against fractures in patients with diabetes?. Calcif Tissue Int 88: 209214

83. Johnell O, Kanis JA, Black DM, Balogh A, Poor G, et al. (2004) Associations between baseline risk factors and vertebral fracture risk in the Multiple Outcomes of Raloxifene Evaluation (MORE) Study. J Bone Miner Res 19: 764772 .

84. Meunier P, Roux C, Seeman E, Ortolani S, Badurski J, et al. (2004) The effects of strontium ranelate on the risk of vertebral fracture in women with postmenopausal osteoporosis. N Engl J Med 350: 459-468.

85. Neer R, Arnaud C, Zanchetta J, Prince R, Gaich G, et al. (2001) Effect of parathyroid hormone (1-34) on fractures and bone mineral density in postmenopausal women with osteoporosis. N Engl J Med 344: 1434-1441.

86. Greenspan S, Bone H, Ettinger M, Hanley D, Lindsay R, et al. (2007) Effect of recombinant human parathyroid hormone (1-84) on vertebral fracture and bone mineral density in postmenopausal women with osteoporosis: a randomized trial. Ann Intern Med 146: 326-339.

87. Anastasilakis A, Goulis DG, Koukoulis G, Kita M, Slavakis A, et al. (2007) Acute and chronic effect of teriparatide on glucose metabolism in women with established osteoporosis. Exp Clin Endocrinol Diabetes 115: 108-111. 